\title{
Homens em Atendimento Psicológico na Atenção Básica em Belém do Pará
}

\section{Men in Psychological Care in Basic Attention of Belém do Pará}

\author{
Adelma do Socorro Gonçalves Pimentel' (orcid.org/0000-0003-0048-4976) \\ Caetano da Providência Santos Diniz² (orcid.org/0000-0002-0619-0664) \\ Kamilly Souza do Vale 3 (orcid.org/0000-0002-7031-2240) \\ Franciélcio Ferreira Belucio4 (orcid.org/0000-0003-2954-9833)
}

\begin{abstract}
Resumo
Este estudo apresenta um panorama das principais queixas declaradas pela clientela masculina ao procurar o serviço de psicologia de um centro de saúde de Belém do Pará, Norte do Brasil, por meio do mapeamento dos atendimentos no período de 2009 a 2013. O método desta pesquisa descritiva consistiu no levantamento de dados produzidos por um psicólogo durante seu atendimento clínico. As principais queixas relacionam-se à (i) ansiedade, (ii) depressão, (iii) violência entre parceiros íntimos, e (iv) outros conflitos interpessoais. Os dados coletados também permitiram constatar que os homens constituíram 41,2\% da clientela, concentraram 46,1\% dos atendimentos realizados, encontravam-se em sua maior parte na faixa etária entre os 20 e 29 anos e tenderam a buscar o serviço espontaneamente. A discussão dos resultados apontou para a necessidade de levar em conta as especificidades da clientela masculina e promover um debate mais amplo acerca da saúde do homem, conferindo-lhe maior visibilidade.
\end{abstract}

Palavras-chave: Serviço de Psicologia. Saúde do homem. Masculinidade. Atenção básica à saúde. Queixas.

\begin{abstract}
1

1 Universidade Federal do Pará, Belém, Brasil. E-mail: adelmapi@ufpa.br.

22 Universidade Federal do Pará, Belém, Brasil. E-mail: caediniz@hotmail.com.

33 Universidade Federal do Pará, Belém, Brasil. E-mail: k.millyvale@gmail.com.

44 Universidade Federal do Pará, Belém, Brasil. E-mail: francielcio@gmail.com.
\end{abstract}

This study presents an overview of the main complaints reported by the male clientele when looking for a psychology service in a health center in Belém do Pará, Northern Brazil, through the mapping of visits in the period from 2009 to 2013. The method of this descriptive research consisted of collecting data produced by a psychologist during his clinical care. The main complaints are related to (i) anxiety, (ii) depression, (ii) violence between intimate partners, and (iv) other interpersonal conflicts. The data collected also revealed that men represent $41.2 \%$ of the 
clientele, concentrate $46.1 \%$ of the consultations performed, were mostly in the age group between 20 and 29 years old and tended to seek the service spontaneously. The discussion of the results pointed to the need to take into account the specificities of the male clientele and foster a debate about the men's health, giving it greater visibility.

Keywords: Psychology Service. Men’s Health. Masculinity. Primary health care. Complaints. 


\section{Introdução}

Este estudo se insere no escopo das pesquisas acerca da saúde do homem brasileiro, cujo objetivo principal foi identificar quais queixas levaram os homens a buscarem o serviço de psicologia em um centro de saúde da rede pública de Belém do Pará, sendo que os resultados permitiram também levantar um breve perfil dos usuários no que se refere ao volume de atendimentos, à faixa etária e às formas de encaminhamento ao serviço.

\section{Considerou-se importante a} investigação do tema, haja vista haver na literatura uma grande lacuna no âmbito das pesquisas, particularmente no que se refere aos serviços psicológicos oferecidos pela rede pública de saúde, de forma que se faz necessário o desenvolvimento de um número maior de estudos na área (Pretto, Langaro \& Santos, 2009). No contexto da realidade brasileira, as investigações acerca da saúde do homem não têm recebido a atenção necessária, não obstante os indicadores, cada vez mais, apontarem para a importância do investimento na área devido aos agravos que essa clientela tem sofrido devido a sua resistência para buscar os serviços (Ministério da Saúde, 2008).

No Brasil, a publicação da Política Nacional de Atenção Integral à Saúde do Homem (Pnaish) pelo Ministério da Saúde (2008) constituiu-se uma das diretrizes para que os profissionais que atuam na atenção básica configurem o atendimento aos homens de maneira mais específica, além de aumentar sua frequência no uso da rede. De acordo com essa política, eles são mais "vulneráveis" às enfermidades, principalmente as crônicas e graves; morrem mais cedo do que as mulheres; não têm o hábito de acessar os serviços; e geralmente adentram-nos pelas portas da média e alta complexidade, ou seja, quando os agravos já estão presentes e avançados, provocando o agravamento da morbidade e um maior custo para o Sistema Único de Saúde - SUS (Ministério da Saúde, 2008; Couto \& Gomes, 2012; Oliveira, Daher, Silva \& Andrade, 2015; Schraiber \& Figueiredo, 2011; Separavich \& Canesqui, 2013; Silva, Bessa, Silva, Souza \& Cerqueira, 2014a; Silva, Dias, Silva \& Dias, 2014c).

Conforme Couto e Gomes (2012), na medida em que a Pnaish elege a atenção básica como foco de ação prioritária, faz-se necessário enfrentar alguns desafios, quais sejam: (i) reconhecer as demandas e necessidades de sua clientela; (ii) garantir recursos estatais suficientes para a implementação de projetos e suas respectivas avaliações; (iii) capacitar gestores e profissionais; (iv) convocar a participação social na produção das ações (debate, planejamento, execução e avaliação); e (v) promover articulações com as diversas redes de atenção do Ministério da Saúde, como a saúde do trabalhador e o combate à violência, entre outros, sempre a partir de uma perspectiva de gênero relacional e transversal, ou seja, em sua interação com raça/etnia, classe social, diferenças de geração, capital cultural, entre outros.

Segundo Rodríguez (2008), os homens adoecem e morrem mais do que a mulher devido a causas como cardiopatia, câncer, problemas respiratórios, acidentes e violência. 
No campo da saúde mental, não obstante a literatura científica internacional demonstrar uma maior frequência de depressão entre mulheres, elas buscam mais frequentemente ajuda do que eles (Bleiberg \& Markowitz, 2009). No caso do suicídio, por exemplo, o qual está frequentemente relacionado à depressão, 79\% dos óbitos são de homens, de acordo com o Boletim Epidemiológico do Ministério da Saúde, sendo também a sua terceira maior causa de morte entre os 15 e 29 anos (Ministério da Saúde, 2017),

Diversos autores têm ainda sinalizado os impactos das questões de gênero para os cuidados em saúde dessa clientela. Para Santos e Nogueira (2011), os papeis a eles atribuídos configuram-se como scripts que regulam suas emoções, práticas e condutas sociais. Assim, a máxima "meninos não choram" ainda se constitui um dos princípios que integram sua sociabilidade e sua subjetivação, tendendo a “esconder" emoções como medo, tristeza, desilusão, culpa, entre outros, ao associar o controle de sua expressividade emocional ao modelo de sujeito dominante que associa razão (e não emoção) à masculinidade.

Entretanto, essa intricada rede de dominação gera prejuízos para ambos os lados. Segundo Kimmel (2008, p. 19, tradução do autor), "Descobrimos que o sexismo não funciona para as mulheres e menos ainda para os homens", pois se ele funciona para os homens enquanto grupo, não funciona para eles individualmente. Se por um lado eles detêm o lugar da força e da resistência, por outro eles tendem a descuidar de si por se considerarem menos susceptíveis às adversidades.
Essas práticas seriam também estimuladas desde cedo, sendo os meninos continuamente confrontados com os padrões de feminilidade que eles deveriam recusar. WelzerLang (2001, p. 462) afirma:

Descrevi como a educação dos meninos nos lugares monossexuados (pátios de colégios, clubes esportivos, cafés..., mas mais globalmente o conjunto de lugares aos quais os homens se atribuem a exclusividade de uso e/ou de presença) estrutura o masculino de maneira paradoxal e inculca nos pequenos homens a ideia de que, para ser um (verdadeiro) homem, eles devem combater os aspectos que poderiam fazê-los serem associados às mulheres.

O gênero, enquanto demarcador social, estabelece padrões de influência da conduta dos indivíduos quanto a ser homem e a ser mulher. Ele funciona como dispositivo social intimamente vinculado às condições da cotidinianidade de forma temporal e mutável (Butler, 2016; Connell \& Messerschmidt, 2013). Segundo Butler (2016, p. 21), “se tornou impossível separar a noção de 'gênero' das interseções políticas e culturais em que invariavelmente ela é produzida e mantida".

As diversas concepções de masculinidades organizam as condutas dos indivíduos em sociedade, sinalizando tanto o que seria próprio de seu sexo quanto o que lhe seria impróprio, como é o caso das condutas atribuídas ao feminino e ao homossexual. A questão do trabalho, por exemplo, de acordo com Rodríguez (2008), está de tal maneira arraigada ao masculino que um homem sem ele dificilmente se sentiria um indivíduo. Ele se sentiria rebaixado não só em sua dignidade ou 
moral como também em sua condição de homem. Sua relação com o mundo do trabalho e da economia é tal que o status por ele gerado pode se tornar avidamente desejado. Arcar com seus gastos, prover o lar e construir um patrimônio pode representar não só independência como também reconhecimento, respeito, autoridade e poder.

Outra concepção refere-se ao exercício da força versus delicadeza. A ele é permitida e socialmente estimulada a expressão da agressividade, seja no ataque, seja na defesa, assim como a prontidão para agir ou reagir às ameaças ou para reunir as energias necessárias à consecução de seus objetivos. A delicadeza não lhe seria típica por ser antagonicamente atribuída ao feminino devido à sua associação com o cuidado e a ternura maternais. Trata-se de um entre os diversos binômios que caracterizam a construção social das masculinidades como sexo-amor, atividade-passividade, razão-emoção, invulnerabilidade-vulnerabilidade, entre outros.

$$
\text { Essa pretensa "superioridade }
$$
masculina" poderia ser também associada à relação do homem com o trabalho, pois a "invulnerabilidade" remete às exigências de uma dedicação intensa aos meios de subsistência, as quais põem com frequência o bem-estar do homem em segundo plano, ficando o cuidado de si subjugado à ideia de estar sempre pronto para o trabalho, apresentando um vigor físico incompatível com a possibilidade de adoecimento (Couto \& Gomes, 2012).

Tais reflexões evidenciam a necessidade de se pensar uma masculinidade que, não obstante tenha diferenças em relação à mulher, não busque reforçá-las apoiando-se nos binarismos de macho e fêmea, provedorcuidadora, forte-frágil (Figueroa-Perea, 2016; Müller, 2012), mas se volte à vivência de uma masculinidade menos dura e mais atenta às suas emoções, a um maior compromisso com a paternidade e a um maior autocuidado, por oposição a uma sociedade cuja influência do machismo causa sérias consequências não só para as relações entre os sexos como para a saúde do próprio homem (Fabbri, 2016).

\section{Método}

Este estudo foi aprovado pelo Comitê de Ética em Pesquisa com Seres Humanos da Universidade Federal do Pará - Instituto de Ciências da Saúde (CAAE n. 38093514.7.0000.0018), que está relacionado com o Parecer n. 893.044 (17 de novembro de 2014), tendo feito parte do projeto maior intitulado "Homens e mulheres em conflito: um estudo acerca da violência conjugal a partir da psicoterapia na atenção básica à saúde”. Depois da obtenção da aprovação do Comitê de Ética, e tendo havido autorização da direção do centro de saúde, deu-se início à coleta de dados por meio do levantamento dos registros dos atendimentos aí realizados pelo serviço de Psicologia. Esse centro faz parte da rede pública do município de Belém do Pará e tem desde 1993 um convênio com a universidade do estado, funcionando tanto como unidade prestadora de serviços quanto como campo de ensino e pesquisa.

O objetivo do levantamento foi identificar quais as queixas que levaram os homens à consulta psicológica, mas, a fim de 
melhor caracterizar a clientela, os dados também possibilitaram observar o número de usuários atendidos e as formas pelas quais eles chegaram ao serviço e faixas etárias.

De caráter descritivo, a pesquisa não envolveu manipulação de variáveis, aplicação de técnicas ou procedimentos em humanos, tendo consistido basicamente na catalogação de prontuários de 419 homens entre um universo de 1.016 usuários atendidos pelo psicólogo no período de 2009 a 2013, cujas identidades foram aqui preservadas a fim de se manter o sigilo próprio da ética em pesquisa.

O levantamento de dados teve como base os atendimentos realizados pelo psicólogo em seu turno (de 6 horas) e foi feito a partir de uma ficha de levantamento pré-elaborada pela equipe de pesquisadores, a qual consistia na coleta das seguintes informações: nome, idade, sexo, número de atendimentos realizados com o usuário e motivo da consulta - esse último transformado em impressões diagnósticas feitas pelo psicólogo ou pelo profissional que fez o encaminhamento durante a consulta, os quais tomavam como base os sinais e sintomas relatados pelo usuário.

A pesquisa documental consistiu de três etapas: (i) seleção dos registros referentes ao período de 2009 a 2013; (ii) identificação do número total de usuários e seu sexo; e (iii) leitura detalhada dos registros dos usuários do sexo masculino a fim de preencher a ficha de coleta de dados. A análise dos resultados foi realizada a partir da leitura das fichas, observando-se a frequência com que determinados aspectos apareciam, destacando-se assim dos demais, o que possibilitou a elaboração de planilhas a fim de melhor visualizá-los. Dispondo-se de tais planilhas, foi possível identificar três categorias: (i) o número de usuários homens atendidos e as formas pelas quais eles chegaram ao serviço; (ii) as faixas etárias que concentraram a maior quantidade de usuários; e (iii) as principais queixas que os levaram a buscar o serviço.

A construção das categorias não foi definida a priori, tendo surgido da coleta de dados, espontaneamente, à medida que se procedia à leitura das fichas, fazendo-se um contraponto com a literatura por meio da reflexão, observando-se semelhanças e diferenças com relação aos autores estudados.

\section{Resultados e Discussão}

\section{O Número de Homens Atendidos e como Chegaram ao Serviço}

No período de 2009 a 2013, foram atendidos 1.016 usuários, dos quais 419 eram homens, o que equivale a $41,2 \%$, os quais concentraram $46,1 \%$ dos atendimentos realizados (Tabela $1)$.

Tabela 1. Atendimentos por gênero

\begin{tabular}{lccccccc}
\hline \multicolumn{1}{c}{ Categorias } & \multicolumn{2}{c}{ Mulheres } & \multicolumn{2}{c}{ Homens } & \multicolumn{2}{c}{ Não identificado } & Total \\
\hline & $\mathrm{N}$ & $\%$ & $\mathrm{~N}$ & $\%$ & $\mathrm{~N}$ & $\%$ & $\mathrm{~N}$ \\
Usuários & 595 & $58,6 \%$ & 419 & $41,2 \%$ & 2 & $0,1 \%$ & 1.016 \\
Atendimentos & 1.609 & $53,7 \%$ & 382 & $46,1 \%$ & 4 & $0,2 \%$ & 2.995 \\
\hline
\end{tabular}

Fonte: Elaborada pelos autores. 
Essa busca pelo serviço pode ser considerado um dado expressivo, já que, como visto, os estudos têm indicado que, tradicionalmente, os homens tendem a procurar os serviços de saúde apenas quando estão afetados por alguma enfermidade, desprezando o caráter preventivo que o cuidado à saúde preconiza, principalmente no que se refere à atenção básica (Schraiber \& Figueiredo, 2011; Silva et al., 2014a)

Outro dado interessante pode ser aqui relacionado a esse possível processo de mudança de mentalidade: a origem da demanda, ou seja, as formas como eles chegaram ao serviço.
Percebe-se que a demanda espontânea (que advém da iniciativa própria ou do incentivo de parentes ou pessoas próximas) foi o meio utilizado por $69,1 \%$ dos usuários na faixa etária de 18 a 59 anos (destacou-se aqui essa faixa, pois as demais tendem a chegar ao serviço por encaminhamento devido a sua própria condição de crianças, adolescentes ou idosos), sendo que o encaminhamento por profissionais e instituições foi o caso de 30,9\% dos usuários (Tabela 2).

Tabela 2. Origem da demanda

\begin{tabular}{lcccccc}
\hline \multicolumn{1}{c}{ Faixa etária } & \multicolumn{2}{c}{$\begin{array}{c}\text { Demanda } \\
\text { espontânea }\end{array}$} & \multicolumn{2}{c}{ Encaminhamento } & Total \\
& $\mathrm{N}$ & $\%$ & $\mathrm{~N}$ & $\%$ & $\mathrm{~N}$ \\
\hline Crianças (0 a 11 anos) & 63 & $42,0 \%$ & 87 & $58,0 \%$ & 150 \\
Adolescentes (12 a 17 anos) & 39 & $67,2 \%$ & 19 & $32,8 \%$ & 58 & 181 \\
Adultos (18 a 59 anos) & 125 & $69,1 \%$ & 56 & $30,9 \%$ & 15 \\
Idosos (igual ou superior a 60 anos) & 5 & $33,3 \%$ & 10 & $66,7 \%$ & 15 \\
Não identificado & 12 & $80,0 \%$ & 3 & $20,0 \%$ & 15 \\
\hline Total & $\mathbf{2 4 4}$ & $\mathbf{5 8 , 2 \%}$ & $\mathbf{1 7 5}$ & $\mathbf{4 1 , 8 \%}$ & $\mathbf{4 1 9}$ \\
\hline
\end{tabular}

Fonte: Elaborada pelos autores.

Esse alto índice de demanda espontânea pode levar à seguinte reflexão: estariam os modelos tradicionais de masculinidade sofrendo mudanças na contemporaneidade? (Beiras, Moraes, Alencar-Rodrigues \& Cantera, 2012; Kimmel, 2008). As ações em saúde tornam-se aqui fundamentais para contribuir na superação da histórica associação entre "homem" e "invulnerabilidade" típica das construções de masculinidade hegemonicamente presentes em nossa cultura (Connell \& Messerschmidt, 2013;
Moura, Santos, Neves, Gomes \& Schwarz, 2014). Ações como campanhas, abordagem de problemas de saúde que o afetam, incentivos à sua participação e assim por diante, ao aproximá-los da atenção básica, contribuem também para desconstruir a ideia de que profissionais como o psicólogo trabalham apenas com pessoas com transtornos mentais. 


\section{As faixas etárias que concentraram a maior quantidade de usuários}

Observando-se a Tabela 3, percebe-se que, no universo dos 419 homens atendidos, a faixa etária que concentrou o maior número de usuários está entre 18 e 59 anos, com 43,2\%. Trata-se das idades mais produtivas para eles, a do jovem e do adulto, as quais guardam suas peculiaridades.

Tabela 3. Faixas etárias dos homens atendidos

\begin{tabular}{ccc}
\hline & \multicolumn{2}{c}{ Homens } \\
Faixas etárias & N & $\mathbf{0}$ \\
\hline Crianças (0 a 11 anos) & 150 & $35,8 \%$ \\
Adolescentes (12 a 17 anos) & 58 & $13,8 \%$ \\
Adultos (18 a 59 anos) & 181 & $43,2 \%$ \\
Idosos ( $\geq$ 60 anos) & 15 & $3,6 \%$ \\
Não identificado & 15 & $3,6 \%$ \\
\hline Total & $\mathbf{4 1 9}$ & $\mathbf{1 0 0 \%}$ \\
\hline
\end{tabular}

Fonte: Elaborada pelos autores.

Detalhando-se de 10 em 10 anos a faixa de 20 a 59 anos, a maior parte dos homens encontra-se na faixa dos 20 aos 29 (36, 1\%), conforme a Tabela 4. Esse dado remete diretamente às queixas que os levaram a buscar o serviço. Conforme se verá adiante nas impressões diagnósticas relatadas na consulta, as queixas que os levaram a procurar atendimento (ansiedade, depressão, violência entre parceiros íntimos e outros conflitos interpessoais) são dificuldades que se relacionam, com frequência, a circunstâncias que são típicas do período da vida que vai dos 20 aos 39 anos, a saber, a intensa preocupação "em ter que" estar social e financeiramente "realizado" (o que pode ser agravado devido à atual instabilidade econômica do País), as deficiências na formação acadêmica e a preparação para o mercado de trabalho, assim como a dificuldade para o planejamento de projetos pessoais (Erikson, 1987).

Tabela 4. Faixas etárias decenais dos usuários de 20 a 59 anos

\begin{tabular}{lcccc}
\hline & \multicolumn{2}{c}{ Mulheres } & \multicolumn{2}{c}{ Homens } \\
\cline { 2 - 5 } \multicolumn{1}{c}{ Faixa etária } & $\mathbf{N}$ & $\mathbf{0}$ & $\mathbf{N}$ & $\mathbf{\%}$ \\
\hline 20 a 29 anos & 89 & $25,1 \%$ & 60 & $36,1 \%$ \\
30 a 39 anos & 94 & $26,5 \%$ & 37 & $22,3 \%$ \\
40 a 49 anos & 89 & $25,1 \%$ & 37 & $22,3 \%$ \\
50 a 59 anos & 83 & $23,4 \%$ & 32 & $19,3 \%$ \\
\hline N total & $\mathbf{3 5 5}$ & & $\mathbf{1 6 6}$ \\
\hline Idade média & $\mathbf{3 9 , 0}$ & & $\mathbf{3 6 , 8}$
\end{tabular}

Fonte: Elaborada pelos autores. 
A visualização dos dados em decênios facilita ainda perceber que, não obstante os homens constituírem $41,2 \%$ do total de usuários atendidos pelo serviço, percebe-se uma significativa diferença entre o número absoluto de homens (166) e de mulheres (355) quando se toma a faixa etária adulta de 20 a 59 anos. Essa visível desigualdade corresponde às análises de Schraiber e Figueiredo (2011, p. 32), segundo as quais os "homens e mulheres são também, perante a saúde, sujeitos desiguais, desde o valor do adoecer às atribuições do cuidado de si e dos outros".

Essa realidade se repete na atenção básica. De acordo com Silva et al. (2014a, p. 77), "Utilizando-se da comparação da presença das mulheres nos serviços de saúde, os homens buscam muito menos os serviços de atenção básica". Tal ausência preocupa por ser a atenção básica a porta de entrada do Sistema Único de Saúde e concentrar as ações preventivas, por apresentar-se como um espaço privilegiado para o desenvolvimento de estratégias capazes de promover o bem-estar e efetivar a integralidade de assistência (Barbosa, 2014; Santos, Rios \& Jesus, 2014).

Mas a ausência dos homens pode ser explicada não só pelo já assinalado fator cultural que desestimula o autocuidado masculino ou pela falta de tempo devido ao trabalho, como também pela femininização dos espaços de saúde (Martins \& Modena, 2015, Pereira et al., 2015). Estes têm se mostrado tipicamente femininos, seja pelos tipos de serviços oferecidos, seja pelas ações em geral voltadas à saúde da mulher, além da ausência de profissionais capacitados para acolher e atender a clientela masculina, fazendo com que eles se sintam deslocados nesse ambiente (Cavalcanti et al., 2014; Pereira et al., 2015; Pereira \& Barros, 2015; Silva et al., 2014c; Siqueira, Oliveira, Mendes, Ximenes \& Moraes, 2014).

\section{As principais queixas que fazem os homens buscarem o serviço}

As queixas declaradas pelos usuários foram agrupadas em 13 grupos, conforme disposto na Tabela 5, entre os quais se destacam os quatro primeiros devido aos altos percentuais alcançados: a ansiedade (37,3\%), a depressão (31,9\%), a violência entre parceiros Íntimos (18,7\%) e conflitos interpessoais

$(16,9 \%)$. 
Tabela 5. Queixas relatadas na consulta psicológica por homens de 20 a 59 anos

\begin{tabular}{lc}
\hline \multicolumn{1}{c}{ Queixas } & $\%$ \\
\hline 1. Ansiedade & $37,3 \%$ \\
2. Depressão & $31,9 \%$ \\
3. Violência entre parceiros íntimos & $18,7 \%$ \\
4. Conflitos interpessoais & $16,9 \%$ \\
5. Somatização & $9 \%$ \\
6. Transtornos do Desenvolvimento & e \\
Aprendizagem & $8,4 \%$ \\
7. Sexualidade & $8,4 \%$ \\
8. Dependência química & $7,8 \%$ \\
9. Esquizofrenia & $5,4 \%$ \\
10. Psicodiagnóstico & $3,6 \%$ \\
11. Agressividade & $3 \%$ \\
12. Transtornos alimentares & $1,2 \%$ \\
13. Queixas não especificadas & $10,2 \%$ \\
\hline \multicolumn{2}{c}{ Fonte: Elaborada pelos autores. }
\end{tabular}

Fonte: Elaborada pelos autores.

A clientela adulta masculina relatou com frequência dois dos principais transtornos relacionados ao sofrimento psicológico na atualidade: a ansiedade e a depressão. De acordo com a American Psychiatric Association (2014), a ansiedade é um dos transtornos mais prevalentes na população em geral, podendo apresentar tanto sintomas físicos (taquicardia, compressão torácica, sudorese, hipoventilação) quanto sintomas psicológicos (medo, tensão, sensação de perigo iminente).

O estresse (37,9\%), a ansiedade $(37,9 \%)$, a síndrome do pânico $(10,6 \%)$, o estresse pós-traumático $(7,6 \%)$, o transtorno obsessivo compulsivo $(4,5 \%)$ e a fobia social $(1,5 \%)$ foram os sintomas referidos no grupo da ansiedade. Os usuários relatavam-nos associando-os a situações como problemas conjugais, ansiedade social e dificuldades financeiras. Tais queixas, considerando-se a faixa-etária de homens adultos jovens, correspondem de fato às preocupações típicas desse período da vida, por exemplo: (i) a busca pelo envolvimento amoroso por meio de vínculos afetivos mais fortes; (ii) a questão da sociabilidade por meio de interações significativas, aceitação social entre outros; e (iii) a necessidade de obter segurança financeira com a realização de projetos de vida, como a conquista de um emprego estável (Erikon, 1987).

Esses resultados confirmam a pesquisa realizada por Pereira e Barros (2015, p. 596) acerca da clientela masculina atendida em um serviço de saúde da rede pública no Piauí. Segundo os autores, "As principais queixas de saúde dos homens estão relacionadas a sintomas agudos e que dificultam as atividades de trabalho, tais como doenças cardiovasculares, hipertensão e diabetes e questões da ordem da sexualidade". 
No grupo da depressão, o indicativo de $37,9 \%$ não é surpreendente, uma vez que, segundo Young, Rygh, Weinberger e Beck (2009, p. 255), ela "é um dos transtornos mais comuns encontrados por profissionais de saúde mental". Os usuários referiram sintomas como "desejos de suicídio" e luto. Esse dado é preocupante por revelar que os homens têm sido acometidos com frequência por sentimentos intensos de tristeza e desânimo, ao contrário do que pensa o senso comum. De acordo com Blay (2014), o padrão de masculinidade hegemônica tende a considerar a tristeza um sinal de fraqueza, o que é transmitido como herança cultural da educação do homem já na infância.

O terceiro grupo refere-se à "Violência entre parceiros íntimos" (VPI), versão portuguesa para a expressão Intimate Partner Violence (Cezario, Fonseca, Lopes \& Lourenço, 2015; Hines, Douglas \& Mccarthy, 2012), e pode, segundo Silva, Coelho e Moretti-Pires (2014b, pp. 278-279), ser definida como “[...] qualquer comportamento no âmbito de um relacionamento íntimo que cause dano físico, moral ou sexual ao companheiro".

Os relatos englobaram conflito conjugal com a parceira íntima (64,5\%), sofrimentos de violência psicológica causada pela companheira $(20,0 \%)$, de violência física causada pela companheira $(2,2 \%)$ e de traição por parte da companheira (2,2\%), além de separação conjugal $(8,9 \%)$ e exercício da violência psicológica conjugal contra a companheira $(2,2 \%)$.

Os estudos acerca da violência conjugal têm constatado que as mulheres tendem a sofrer mais violência física por parte dos seus parceiros, enquanto estes indicam sofrer mais violência psicológica, como foi observado nas revisões de literatura dos últimos 15 anos feitas por Machado e Matos (2014), Silva et al. (2014a) e Grossi, Minella e Losso (2006), de estudos como os de Alvim e Souza (2005) e Martins (2008). Esse tipo de violência foi por eles descrito como humilhação pública, intimidação, controle, questionamento da masculinidade, ameaças, perseguição, entre outros, o que corresponde aos resultados de diversos estudos (Hines \& Douglas, 2011; Silva et al., 2014b). Hines e Douglas (2009, p. 575, tradução do autor), por exemplo, afirmam que "Relatórios sobre a prevalência de agressão psicológica da mulher contra o homem estimam que pelo menos metade, e até mesmo $90 \%$, dos homens sofrem algum tipo de ato psicologicamente agressivo (exemplo, ser ameaçado, xingado, ou ser insultado) em seus relacionamentos". Vale observar que não se trata de afirmar que os homens não cometam esse tipo violência contra suas parceiras, e sim de constatar que entre os relatos de homens e mulheres são eles os que afirmam sofrer mais frequentemente.

Seus relatos foram considerados particularmente interessantes, já que raramente eles conseguem falar sobre esse assunto com seus pares devido ao receio de serem criticados (Barber, 2008; Shuler, 2010), podendo-se por isso dizer que eles consideraram o atendimento psicológico um momento propício para falar do assunto sem temer serem julgados.

O quarto grupo, conflitos interpessoais, inclui as dificuldades vivenciadas nas habilidades sociais e nas relações humanas em geral, assim como nos conflitos familiares, tendo um 
destaque maior a relação com os pais. Os indicadores foram distribuídos entre dificuldade de interação social $(50,0 \%)$, dependência da figura materna $(33,3 \%)$, baixa autoestima $(8,3 \%)$, timidez $(5,6 \%)$ e isolamento social $(2,8 \%)$, o que aponta para a necessidade de trabalhar aspectos como a autoexpressão, a resolução de conflitos sem o uso da força, assim por diante - esses últimos diretamente relacionados às mudanças nos estereótipos de masculinidade dominantes na sociedade. Aqui, mais uma vez, considera-se que políticas públicas e ações em saúde podem contribuir significativamente para a qualidade de vida, sendo importante que os profissionais sejam capacitados para lidar com tais questões, gerando impacto tanto em si mesmos, suas próprias convicções, seus próprios conflitos e preconceitos, quanto nos seus trabalhos no cotidiano de assistência à clientela masculina que a eles recorrem (Carneiro, Santos, Macena \& Vasconcelos, 2016).

\section{Considerações finais}

Esta pesquisa possibilitou refletir sobre a importância de se atentar para as especificidades da clientela masculina na atenção básica, particularmente no que se refere às suas demandas de atendimento psicológico. Além das questões culturais, estão relacionados fatores como os horários de trabalho, o que acaba sendo empecilho para muitos homens buscarem os serviços de saúde, de forma que se faz necessário o envolvimento das relações trabalhistas no sentido de sensibilizar empregados e patrões para a saúde do homem, facilitando a eles o acesso aos serviços e o fomento do autocuidado.

As ações preventivas deveriam ultrapassar os moldes dos atendimentos tradicionais que ocorrem dentro dos muros do centro de saúde e perguntar: que outras estratégias podem ser utilizadas para alcançar esta população? Como se aproximar desses homens sem esperar que eles venham ao centro? E uma vez no centro, como trabalhar com eles? Seria o trabalho em grupo uma forma mais eficaz de reelaboração dos modelos de masculinidades, de obtenção de apoio e de socialização? Como reorganizar os espaços para acolhê-los? De que forma a comunidade poderia contribuir para essa necessária mudança de mentalidade? São questões para serem aprofundadas em outros estudos. Acredita-se que as respostas a tais questões poderiam contribuir para o oferecimento de uma atenção mais voltada para as suas necessidades, provocando impactos positivos sobre sua qualidade de vida.

Quanto às limitações do estudo, a pesquisa requer maior aprofundamento com relação às queixas de homens mais jovens, na faixa etária de 20 a 29 anos, já que o estudo apresenta os resultados das respostas de todas as faixas etárias e não especificamente a esta, que concentrou o maior número de usuários adultos. Outro ponto refere-se à necessidade de aprofundar o perfil da clientela a fim de enriquecer os dados, por exemplo, o perfil socioeconômico, a escolaridade, assim por diante. Por fim, ainda que este estudo tenha focalizado a chegada do homem ao serviço e, portanto, a consulta psicológica, seria 
interessante saber como se deu o seguimento do atendimento, avaliando-se o nível de adesão dos usuários ao acompanhamento.

\section{Referências}

Alvim, S., \& Souza, L. (2005). Violência conjugal em uma perspectiva relacional: homens e mulheres agredidos/agressores. Psicologia: Teoria e Prática, 7(2), 171- 206. Doi 10.1590/S0102-37722008000200006.

American Psychological Association (2014). Intimate Partner Abuse and Relationship Violence. Washington, DC: APA. Retrieved September 2, 2016, from http://www.apa.org/pi/iparv.pdf

Barber, C. F. (2008). Violence Domestic against Men. Nursing Standard, 22(51), 35-39. Retrieved March 23, 2016, from http://journals.rcni.com/doi/pdfplus /10.7748/ns2008.08.22.51.35.c6644.

Barbosa, C. J. M. (2014). Saúde do homem na atenção primária: mudanças necessárias no modelo de atenção. Saúde $e$ Desenvolvimento, 6(3), 99-114. Recuperado em 2 abril, 2017, de https://www.uninter.com/revistasaud e/index.php/saudeDesenvolvimento/ article/view/277/226.

Beiras, A., Moraes, M., Alencar-Rodrigues, R. de, \& Cantera, L. (2012). Políticas e leis sobre violência de gênero: reflexões críticas. Revista Psicologia e Sociedade, 24(1), 36-45. Recuperado em 15 março, 2016, de

http://www.scielo.br/pdf/psoc/v24n 1/05.pdf.

Blay, E. A. (2014). Feminismos e masculinidades: novos caminhos para enfrentar a violência contra a mulher. São Paulo: Cultura Acadêmica.

Bleiberg, K., \& Markowitz, J. (2009). Psicoterapia interpessoal para depressão. In D. Barlow et al. (Eds.). Manual clínico dos transtornos psicológicos: tratamento passo a passo (pp. 309-328). Porto Alegre: Artmed.
Butler, J. (2016). Problemas de gênero: feminismo e subversão de identidade (11a ed.). Rio de janeiro: Civilização Brasileira.

Carneiro, L. M. R., Santos, M. P. A. dos, Macena, R. H. M., \& Vasconcelos, T. B. de (2016). Atenção integral à saúde do homem: desafio na atenção básica. Revista Brasileira de Promoção à Saúde, 29(4), 554563. Recuperado em 23 setembro, 2017, de http://periodicos.unifor.br/RBPS/art icle/view/5301/pdf.

Cavalcanti, J. da R. D., Ferreira, J. de A., Henriques, A. H. B., Morais, G. S. da N., Trigueiro, J. V. S., \& Torquato, I. M. B. (2014). Atenção integral à saúde do homem: necessidades, obstáculos e estratégias de enfrentamento. Revista de Enfermagem Escola Ana Nery, 18(4), 628$634 . \quad$ Doi: $\quad 10.5935 / 1414-$ 8145.20140089.

Cezario, A. C. F., Fonseca, D. S., Lopes, N. C., \& Lourenço, L. M. (2015). Violência entre parceiros íntimos: uma comparação dos índices em relacionamentos hetero e homossexuais. Temas em Psicologia, 23(3), 565-575. Doi: 10.9788/Tp2015.3-04.

Connell, R. W., \& Messerschmidt, J. W. (2013). Masculinidade hegemônica: repensando o conceito. Revista Estudos Feministas, 21(1), 241-282. Recuperado em 22 março, 2017, de

http://www.scielo.br/pdf/ref/v21n1 /14.pdf.

Couto, M. T., \& Gomes, R. (2012). Homens, saúde e políticas públicas: a equidade de gênero em questão. Ciência \& Saúde Coletiva, 17(10), 2569-2578. Recuperado em 28 dezembro, 2016, de http://www.scielo.br/pdf/csc/v17n1 0/02.pdf.

Erikson, E. (1987). Infância e sociedade (2a ed.). Rio de janeiro: Zahar.

Fabbri, L. (2016). Colectivos de hombres y feminismos. Aportes, tensiones y desafíos desde (y para) la praxis. Sexualidad, Saludy Sociedad, 22, 355-368. Doi: 10.1590/1984-6487.sess.2016.22.16.a. 
Figueroa-Perea, J.-G. (2016). Algunas reflexiones para dialogar sobre el patriarcado desde el estudio y el trabajo con varones y masculinidades. Sexualidad, Salud y Sociedad, 22, 221-248. Doi: 10.1590/1984-6487.Sess.2016.22.10.A.

Grossi, M., Minella, L. S., \& Losso, J. C. M. (2006). Gênero e violência, pesquisas acadêmicas brasileiras (1975-2005). Florianópolis: Mulheres.

Hines, D. A., \& Douglas, E. M. (2009). Women's Use of Intimate Partner Violence against Men: Prevalence, Implications and Consequences. Journal of Aggression, Maltreatment \& Trauma, 18, 572-586.

Doi: 10.1080/10926770903103099.

Hines, D., \& Douglas, E. (2011). Symptoms of Post-traumatic Stress Disorder in Men who Sustain Intimate Partner Violence: a Study of Help Seeking and Community Samples. Psychology of Men \& Masculinity, 12, 112-127. Retrieved from http://dx.doi.org/10.1037/a0022983.

Hines, D. A., Douglas, E. M., \& McCarthy, S. C. (2012). Men who Sustain Female-toMale Partner Violence: Factors Associated with where they Seek Help and how they Rate those Resources. Violence Victims, 27(6), 871-894. Doi:10.1891/0886-6708.27.6.871.

Kimmel, M. (2008). Los estudios de la masculinidad: una introducción. In À. Carabí \& J. M. Armengol (Orgs.). La masculinidad a debate (pp. 15-31). Barcelona, Icaria.

Machado, A., \& Matos, M. (2014). Homens vítimas na intimidade: análise metodológica dos estudos de prevalência. Revista Psicologia e Sociedade, 26(3), 726-736. Recuperado em 20 outubro, 2015, de http://www.scielo.br/pdf/psoc/v26n 3/a21v26n3.pdf.

Martins, G. A. (2008). Estudo de caso: uma estratégia de pesquisa. São Paulo: Atlas.

Martins, A. M., \& Modena, C. M. (2015). A saúde do homem nos serviços de atenção primária: desafios culturais e organizacionais. Cadernos da Escola de Saúde Pública do Ceará, 9(20), 40-52. Recuperado em 22 outubro, 2016, de http://www.esp.ce.gov.br/cadernoses $\mathrm{p} /$ index.php/cadernosesp/article/vie $\mathrm{w} / 368 / 122$.

Ministério da Saúde. Secretaria de Atenção à saúde. Departamento de Ações Programáticas Estratégicas (2008). Política Nacional de Atenção integral à saúde do homem: princípios e diretrizes. Brasília, DF: Autor.

Ministério da Saúde. Secretaria de Vigilância em Saúde (2017). Boletim epidemiológico das tentativas e óbitos por suicídio no Brasil e a rede de atenção à saúde. 48(30). Brasília: Autor.

Moura, E. C. de, Santos, W. dos, Neves, A. C. M. das, Gomes, R., \& Schwarz, E. (2014). Atenção à saúde dos homens no âmbito da Estratégia Saúde da Família. Ciência \& Saúde Coletiva, 19(2), 429-438. Doi: $10.1590 / 1413$ $\underline{81232014192.05802013 .}$

Müller, R. F. (2012). Violência, vulnerabilidade e risco na política nacional de atenção integral à saúde do homem. Revista EPOS, 3(2), 1-15. Recuperado em 14 outubro, 2016, de http://revistaepos.org/arquivos/06/ RitaFlores.pdf.

Oliveira, M. M. de, Daher, D. V., Silva, J. L. L. da, \& Andrade, S. S. C. A. (2015). A saúde do homem em questão: busca por atendimento por atendimento na atenção básica de saúde. Ciência \& saúde coletiva, 20(1), 273-278. Doi:10.1590/141381232014201.21732013.

Pereira, M. C. A., \& Barros, J. P. P. (2015). Públicos masculinos na Estratégia de Saúde da Família: estudo qualitativo em Parnaíba-PI. Psicologia \& Sociedade, 27(3), 587-598. Doi:10.1590/1807$03102015 \mathrm{v} 27 \mathrm{n} 3 \mathrm{p} 587$.

Pereira, M. M. M., Cézar, E. S. R., Pereira, V. C. L. da S., Braga, L. S., Espínola, L. L., \& Azevedo, E. B. (2015). Saúde do homem na atenção básica: análise acerca do perfil e agravos à saúde. Revista de Enfermagem, 
9(1), 440-447. Doi:10.5205/reuol.522143270-1-RV.0901supl201524.

Pretto, Z., Langaro, F., \& Santos, G. (2009). Psicologia Clínica existencialista na atenção básica à saúde. Revista Psicologia, Ciência e Profissão, 29(2), 394-405. Doi:10.1590/S1414$\underline{98932009000200014}$.

Rodríguez, J. C. R. (2008). Ejes estructurales y temáticos de análisis del género de los hombres. Una aproximación. In J. C. R. Rodríguez \& G. U. Vázquez (Orgs.). Masculinidades: el juego de género de los hombres en el que participan las mujeres (pp. 85-112). Madrid: Plaza y Valdés.

Santos, W. T., M, Rios, A. F., \& Jesus, D. S. (2014). Desafios e potencialidades da inserção da Psicologia na atenção básica em saúde. In M. K. B. Souza \& J. S. C. Tavares (Orgs.). Temas em saúde coletiva: gestão e atenção no SUS em debate (pp. 235258). Cruz das Almas, BA: UFRB.

Santos, L., \& Nogueira, C. (2011). Sexualidades masculinas, expressão emocional e afectiva: das (im)possibilidades construídas, às experiências de opressão. In A. I. Sani (Org.). Temas de vitimologia: realidades emergentes na vitimação e respostas sociais (pp. 115-134). Coimbra: Almedina.

Schraiber, L. B., \& Figueiredo, W. S. (2011). Integralidade em saúde e os homens na perspectiva relacional de gênero. In $\mathrm{R}$. Gomes (Org.). Saúde do homem em debate (pp. 19-38). Rio de Janeiro: Fiocruz.

Separavich, M. C., \& Canesqui, A. M. (2013). Saúde do homem e masculinidades na Política de Atenção Integral à Saúde do Homem: uma revisão bibliográfica. Saúde e Sociedade, 22(2), 415-428. Recuperado de http://dx.doi.org/10.1590/S010412902013000200013.

Shuler, C. (2010). Male Victims of Intimate Partner Violence in the United States: um Examination of the Reviews of Literature through Critical Theoretical Perspective. International Journal of Criminal Justice Sciences, 5(1), 163-173. Recuperado em 28 setembro, 2017 , http://www.sascv.org/ijcjs/pdfs/caro lettaijcjs2010vol5iss1.pdf.

Silva, A. A. M. R., Bessa, A. M., Silva, E. A. L., Souza, F. O., \& Cerqueira, T. M. S. (2014a). Atenção à saúde do homem nas unidades de saúde da família. In M. K. B. Souza \& J. S. C. Tavares (Orgs.). Temas em saúde coletiva: gestão e atenção no SUS em debate (pp. 75-103). Bahia, BA: UFRB.

Silva, A. C. L. G., Coelho, E. B. S., \& MorettiPires, R. O. (2014b). O que se sabe sobre o homem autor de violência contra a parceira íntima: uma revisão sistemática. Revista Panam Salud Publica, 35(4), 278283. Recuperado em 12 julho, 2016, de http://www.scielosp.org/pdf/rpsp/v 35n4/07.pdf.

Silva, A. N. da, Dias, M. de P., Silva, D. A. da, \& Dias, L. de P. (2014c). Promoção da saúde do homem nos serviços de atenção primária à saúde. Em extensão, 13(1), 8288. Recuperado em 17 março, 2016, de http://docplayer.com.br/29607161Promocao-da-saude-do-homem-nosservicos-de-atencao-primaria-asaude.html.

Siqueira, E. L., Oliveira, G. R., Mendes, J. D., Ximenes, J. M., \& Moraes, K. M. (2014). Atenção à saúde do homem: trabalhando a percepção do profissional enfermeiro na estratégia saúde da família. Sanare, 13(1), 48-55. Recuperado em 12 junho, 2017, de https://sanare.emnuvens.com.br/san are/article/viewFile/432/287.

Young, J., Rygh, J., Weinberger, A., \& Beck, A. (2009). Terapia cognitiva para depressão. In D. Barlow et al. (Orgs.). Manual clínico dos transtornos psicológicos: tratamento passo a passo (pp. 255-308). Porto Alegre: Artmed.

Welzer-Lang, D. (2001). A construção do masculino: a dominação das mulheres e homofobia. Estudos Feministas, 9(2), 460482. Recuperado em 12 dezembro, 2015, de

http://www.scielo.br/scielo.php?pid $=\mathrm{S} 0104$ 026X2001000200008\&script $=$ sci_abst ract\&tlng $=$ pt. 
Recebido em: 28/2/2018

Aprovado em: 6/9/2018 